\title{
Surrogate Assisted Teaching Learning Based Optimisation for Process Design of a Non-circular Drawing Sequence
}

\author{
N. Pholdee ${ }^{1}$, S. Bureerat ${ }^{1}$, H. M. Baek², \&Y.-T. Im²,3,* \\ ${ }^{1}$ Sustainable Infrastructure Research and Development Center, Department of Mechanical Engineering, Faculty of \\ Engineering, KhonKaen University, Thailand 40002 \\ ${ }^{2}$ National Research Laboratory for Computer Aided Materials Processing, Department of Mechanical Engineering, KAIST, \\ Republic of Korea \\ ${ }^{3}$ Office of the President, KIMM, Republic of Korea \\ * Corresponding Author
}

\begin{abstract}
In this study, surrogate assisted teaching learning based optimisation was conducted for designing a non-circular drawing (NCD) sequence in order to improve the deformation homogeneity of the drawn wire. The objective function was introduced to minimise inhomogeneous distribution of effective strain atthe cross-section of the drawn wire by selecting the design variables such as major to minor axes ratio, semi-die angle, and reduction ofarea. Three surrogate models were used to predict the effective strains atthe crosssection of the drawn wire while the teaching learning based optimiser was used to obtain the optimum results. Finite element analysis was employed for simulation of the NCD sequence in order to determine accurate effective strain distribution. The accuracy of all surrogate models was investigated while optimum results were compared with the previous studies available in the literature. The optimum results found in the present study showed better effective strain homogeneity at the cross-section of the drawn wire with the same total reduction of the area available in the literature for the less number of passes.
\end{abstract}

Keywords-non-circular drawing sequence; surrogate model; evolutionary algorithm; optimal lat in hypercube sampling.

\section{INTRODUCTION}

Steel wires are products of a wire drawing (WD) process, which is widely used in various engineering applications. In the steel wire manufacturing, deformation inhomogeneity between the surface and centre regions of the wires is one of the major issues affecting mechanical properties of the drawn wires. Generally, a design parameter of a conventional WD process is limited to only a semi-die angle

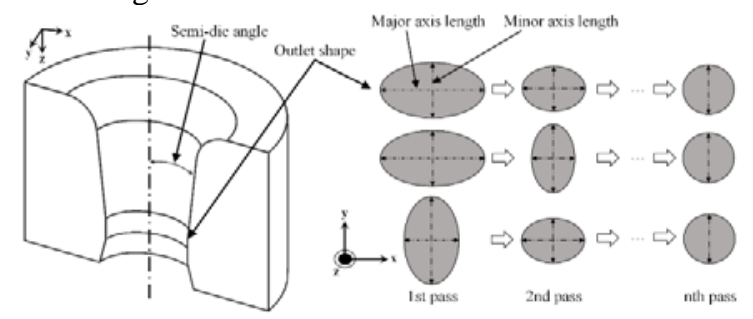

Figure 1. Non-circular shaped die used in the NCD sequence and examples of the possible combination of the NCD sequence.
Since the area of reduction usually set to be constant while the dies shape is usually circular. This makes the WD have some limitations since increasing the semi-die angle leads to the increase of inhomogeneous deformation of the wire. On the other hand, decreasing the semi-die angle leads to the decreasing of the wire strength [1]. Therefore, developing new wire manufacturing process to improve mechanical properties of the drawn wires by imposing relatively homogeneous deformation on the wire compared to the conventional WD is beneficial.

In this regard, a non-circular drawing (NCD) sequence was proposed by Hwang et al. [2] and applied to low carbon steel up to 10 passes of the NCD sequence by Lee et al. [3]. Then, Baek et al. [4] introduced two processing routes for the NCD sequence and applied up to 12th pass for pearlitic steel wire. All of these work showed better strength and ductility compared to one of the conventional WD by imposing relatively high and homogeneous deformation on the wire during the process.

However, processing parameters of the NCD sequence such as major to minor axes ratio, semi-die angle, and reduction of area used in the previous works were set to be constant. Thus, it might be of interest to find optimum values of such processing parameters for further improvement of the NCD sequence.

In this work, surrogate-assisted teaching learning based optimisation for numerically designing the NCD sequence was conducted. The objective function was assigned to minimise the deformation inhomogeneity of the drawn wire by selecting the design variables such as major to minor axes ratio, semi-die angle, and reduction of area. In order to reduce the computational time for the process optimisation of the NCD sequence, the surrogate models were used to predict effective strain distributions at the cross-section of the drawn wire for calculating the objective function of deformation inhomogeneity. Teaching learning based algorithm [5] was used as an optimiser while three well known surrogate models such as polynomial regression model [6], radial basis function [7] model, and Kriging model [8] were employed for comparison. The most efficient surrogate model for the NCD optimisation problem 
will be obtained and the optimum results will be compared to the best results available in the literature.

\section{FORMULATION OF OPTIMISATION DESIGN PROBLEM OF THE NCD SEQUENCE}

Optimisation of the NCD sequence was carried out in order to find the optimal processing parameters including the reduction of area and die geometry for each pass. Figure 1 shows a non-circular shaped die used for the NCD sequence and several examples of the possible sequence combinations of the NCD.

The design variables considered were the reduction of area $(R A)$, axes ratio $(r)$, semi-die angle for each pass $(\theta)$. The reduction ofarea and axes ratio can be expressed as:

$$
\begin{gathered}
R A_{i}=\frac{A_{i-1}-A_{i}}{A_{i-1}}=\frac{\pi a_{i-1} b_{i-1}-\pi a_{i} b_{i}}{\pi a_{i-1} b_{i-1}} \\
r_{i}=\frac{a_{i}}{b_{i}}
\end{gathered}
$$

where $a_{\mathrm{i}}$ and $b_{\mathrm{i}}$ are the major and minor axis lengths of die number $i$. With the given values of the reduction of area and axes ratio, $a_{\mathrm{i}}$ and $b_{\mathrm{i}}$ can be computed from Eqs. 1 and 2 .

The objective function is to minimize the inhomogeneity of the effective strain distributions at the cross-section of the drawn wire. The total number of passes (npass), total reduction of area $\left(R A_{\text {total }}\right)$, and final diameter of the wire were constrained to be $11,0.93$, and $3.41 \mathrm{~mm}$, in that order. Then, the objective function can be expressed as follows:

Algorithm 1Modification of the axes ratio to avoid the under filling during the numerical simulation of the NCD sequence.

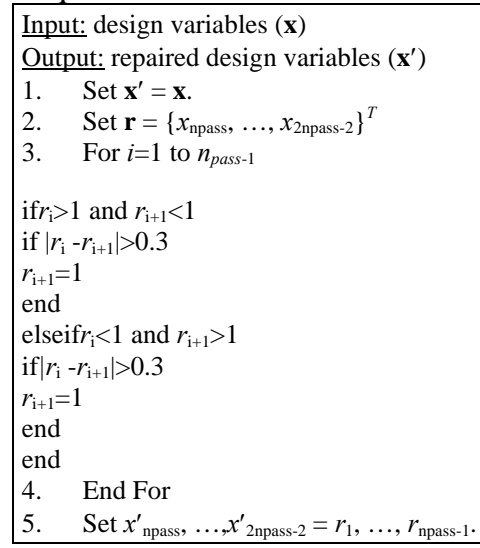

$$
\text { Min: } f(\mathbf{x})=I F=\frac{\operatorname{STD}(\bar{\varepsilon})}{\operatorname{MEAN}(\bar{\varepsilon})}
$$

Subject to:

$$
\begin{gathered}
0<R A_{\text {final }} \leq 0.30 \\
0.15 \leq R A_{i} \leq 0.25 \quad i=1, \ldots, \text { npass }-1 \\
\left|R A_{i}-R A_{i-1}\right| \leq 0.2 \quad i=1, \ldots, \text { npass } \\
0.702 \leq r_{i} \leq 1.425 \\
3 \leq \theta_{i} \leq 10 \text { degree } i=1, \ldots, \text {, npass }
\end{gathered}
$$

where $\mathbf{x}=\left[R A_{1}, \ldots, R A_{\text {npass }-1}, r_{1}, \ldots, r_{\text {npass }-1}, \theta_{1}, \ldots, \theta_{\text {npass }}\right]^{T}$ is a vector of design variables and $\bar{\varepsilon}$ is the effective strain at the cross-section of the drawn wire. $I F$ is an inhomogeneity factor which can be defined as the standard deviation (STD) divided by the mean value of the effective strains [9] at the cross-section of the drawn wire. The $R A_{\text {final }}, a_{\text {final }}$,and $b_{\text {final }}$ are the reduction of area, major axis and minor axis lengths of the final die,in that order. In addition, the "under filling" phenomenon which might cause irregular surface roundness in the drawn wire after the process was also taken into account in this study. One of such casesoccurs when a sudden change of the axes ratio between two successive dies happens, swapping the positions of the major and minor axes.In this case, Algorithm 1 was applied to check and modify axes ratio before performing function evaluation in order to avoid the under filling. However, this algorithm cannotguarantee to avoid the under filling perfectly because of complex factors such as deformation behaviour of the material, die geometry, and processing parameters entwined with the under filling. Therefore, the under filling will be checked againduring the optimum process.

In this study, finite element analysis (FEA) for the NCD sequence was carried out using CAMPform $3 \mathrm{D}$, developed by Kim and Im [10]. Three-dimensional FEA model of one quarters of the work piece with increasing density of the mesh system along the drawing direction similar to the previous work [4] was used in this study. Since each simulation for the NCD sequences is time-consuming, a surrogate model was introduced for the optimisation process by approximating the effective strains for twentyone points, as shown in Fig. 2. Then, the objective function can be calculated from these predicted strain values for the twenty one points based on Eq. 3 .

The common process for surrogate assisted optimisation can be performed in such a way that sampling points are initially generated throughout design domain by using design of experiment (DOE) method. Then, the objective function values of all sampling points will be calculated based on the numerical simulation or actual function evaluation. Thereafter, the model for estimating an objective function will be constructed and the optimisation based on the surrogate model will be performed. Finally, the actual objective function of the optimal result obtained will be calculated.

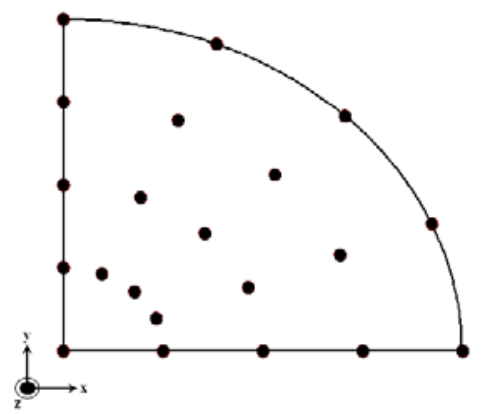

Figure 2. Twenty-one reference points at the cross-section of the drawn wire used to measure inhomogeneity of effective strain. 
In this study, polynomial regression model (PR), radial basis function model (RBF) and Kriging model (KG) were used as surrogate models while the optimal Latin hyper cube sampling technique proposed in [11] was used to generate sampling points. Sixty two sampling points for 31 total number of design variables were generated and used for constructing the surrogate models. Teaching learning based algorithm was used as an optimiser while optimisation parameters likethe population size and the number of generation were set to be 100 and 300, respectively. In addition, the fuzzy penalty function technique was used to handle constraints.

\section{RESULTS AND DISCUSSION}

Having performed the optimization of the NCD sequence described in Eq. 3 based on the three surrogate models, the actual values of the effective strains at the reference points (refer to Fig. 2) of the optimal results were calculated by using FEA. For an optimum solution, the total percentage errorsfor all the reference points at the crosssection of the drawn wire were used to measure the accuracy of the models, and Table 1 shows actual objective function values of the optimum points obtained from the various surrogate models. Figure 3 shows boxplots of the percentage errors obtained from the various surrogate models, and the KG was the most accurate model while the second and the third accurate models were RBF and PR, respectively. From Table 1, the under filling phenomena were checked and the results were also indicated in this table. The feasible solution with lower objective function value was the better optimum design. The optimum results obtained from the RBF was the best. Based on the results in Fig. 3 and Table 1, it should be noted that, although the KG was said to be accurate, it cannot guarantee the optimum solution. This is due to the so-called the blessing and curse of uncertainty, as stated in reference [12].

Table 2 shows the optimal results obtained from the RBF while Fig. 4 shows comparative standard deviation (STD) of effective strains at the cross-section of the drawn wire after the 11th pass for the RBF, the 12th pass for the conventional WD, and the best NCD obtained from the previous work [4]. The results indicate

TABLE I. OPTIMUM RESULTS OBTAINED FROM THE THREE SURROGATE MODELS.

\begin{tabular}{ccc}
\hline Models & $\begin{array}{c}\text { Actual objective function value } \\
(I F)\end{array}$ & $\begin{array}{c}\text { Underfilling } \\
\text { detected }\end{array}$ \\
\hline PRS & 0.0375 & Yes \\
RBF & 0.0342 & No \\
KG & 0.0634 & No \\
\hline
\end{tabular}

TABLE II. PROCESSING PARAMETERS OF THE CONVENTIONAL WD AND NCD IN THE LITERATURE [4] AND OPTIMUM VALUES OBTAINED FROM THE PRESENT WORK.

\begin{tabular}{|c|c|c|c|c|c|c|c|c|c|c|c|c|}
\hline \multirow[b]{2}{*}{$\begin{array}{l}\mathscr{U} \\
\tilde{W} \\
\tilde{0} \\
\stackrel{0}{0} \\
0 \\
\dot{0} \\
\dot{z}\end{array}$} & \multicolumn{3}{|c|}{ Major axis length (mm) } & \multicolumn{3}{|c|}{ Minor axis length (mm) } & \multicolumn{2}{|c|}{$\begin{array}{c}\text { Semi-die angle } \\
\text { (degree) }\end{array}$} & \multicolumn{2}{|c|}{\begin{tabular}{|c|}
$\begin{array}{c}\text { Reduction of area per } \\
\text { each pass }\end{array}$ \\
\end{tabular}} & \multicolumn{2}{|c|}{$\begin{array}{c}\text { Total reduction of } \\
\text { area }\end{array}$} \\
\hline & $\frac{F}{3}$ & $\begin{array}{l}\text { J } \\
己 \\
己\end{array}$ & 贡 & $\frac{F}{3}$ & $\begin{array}{l}\underset{\Xi}{\Xi} \\
z\end{array}$ & 㠭 & 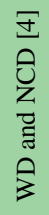 & $\stackrel{\Gamma}{\underline{D}}$ & 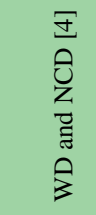 & 步 & 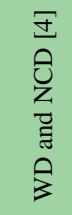 & 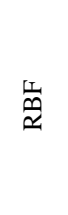 \\
\hline 1 & 11.63 & 13.39 & 9.43 & 11.63 & 10.10 & 13.44 & 6 & 9.93 & 0.20 & 0.25 & 0.20 & 0.25 \\
\hline 2 & 10.40 & 10.40 & 8.63 & 10.40 & 10.40 & 12.21 & 6 & 7.45 & 0.20 & 0.17 & 0.36 & 0.38 \\
\hline 3 & 9.30 & 8.08 & 7.51 & 9.30 & 10.71 & 10.70 & 6 & 3.92 & 0.20 & 0.24 & 0.49 & 0.52 \\
\hline 4 & 8.32 & 8.32 & 6.50 & 8.32 & 8.32 & 9.27 & 6 & 9.98 & 0.20 & 0.25 & 0.59 & 0.64 \\
\hline 5 & 7.44 & 8.57 & 6.66 & 7.44 & 6.46 & 6.79 & 6 & 9.98 & 0.20 & 0.25 & 0.67 & 0.73 \\
\hline 6 & 6.66 & 6.66 & 4.93 & 6.66 & 6.66 & 6.88 & 6 & 3.01 & 0.20 & 0.25 & 0.74 & 0.80 \\
\hline 7 & 5.95 & 5.16 & 4.90 & 5.95 & 6.86 & 5.88 & 6 & 9.81 & 0.20 & 0.15 & 0.79 & 0.83 \\
\hline 8 & 5.32 & 5.32 & 4.72 & 5.32 & 5.32 & 5.17 & 6 & 9.94 & 0.20 & 0.15 & 0.83 & 0.86 \\
\hline 9 & 4.76 & 4.13 & 4.37 & 4.76 & 5.48 & 4.75 & 6 & 3.16 & 0.20 & 0.15 & 0.87 & 0.88 \\
\hline 10 & 4.26 & 4.26 & 3.31 & 4.26 & 4.26 & 4.71 & 6 & 3.00 & 0.20 & 0.25 & 0.89 & 0.91 \\
\hline 11 & 3.81 & 3.31 & 3.41 & 3.81 & 4.39 & 3.41 & 6 & 3.00 & 0.20 & 0.25 & 0.91 & 0.93 \\
\hline 12 & 3.41 & 3.41 & - & 3.41 & 3.41 & - & 6 & - & 0.20 & - & 0.93 & - \\
\hline
\end{tabular}




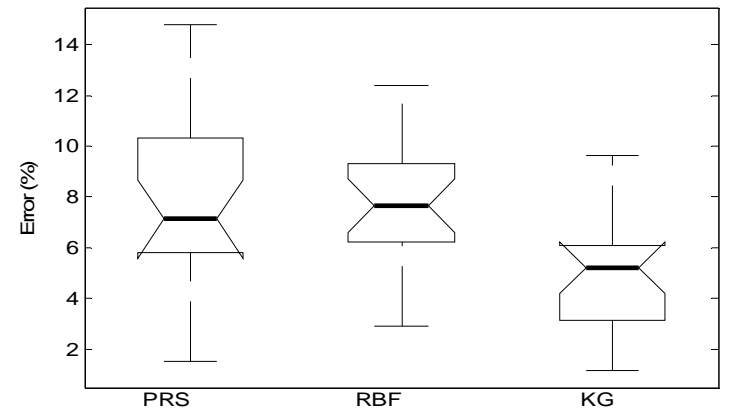

Figure 3. Percentage errors at optimum points of all twenty-one reference points at thecross-section of the drawn wire.

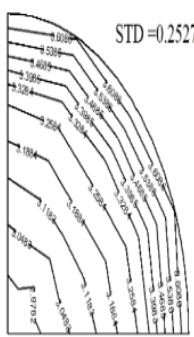

WD

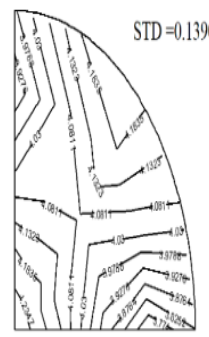

NCD best obtained in $|4|$

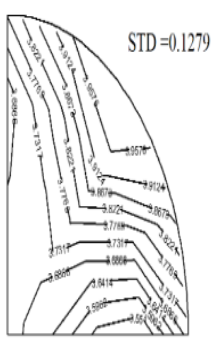

RBF in this study
Figure 4. Comparison of the deformation in homogeneity of the effective strain at the cross-section of the drawn wire.

That within the smaller number of passes and the same total reduction of area, the optimum solutions obtained from the RBF in this study give better deformation homogeneity than the conventional WD and the best NCD obtained from the previous work [4].

\section{CONCLUSIONS}

An optimisation of the NCD sequence based of surrogate-assisted teaching learning based optimisation has been conducted in order to improve the mechanical properties of the drawn wires. The objective function was set to minimise the inhomogeneity of effective strain distribution at the cross-section of the drawn wire.Three surrogate models with the teaching learning based optimiserwere successfully used to obtain the optimum results. The results show that the KG was the best for function estimation while the best optimum results were obtained from the RBF model.When comparing with the previous results available in reference, it was found that within the smaller total number of passes and the same total reduction of area, the optimum solutions obtained from RBF in this study give better effective strain homogeneity than the conventional WD and the best NCD obtained from the previous work.

\section{ACKNOWLEDGEMENTS}

The research work was supported by Thailand Research Funds (TRF) and POSCO.

\section{REFERENCES}

1] Castro,A.L.R.,Campos, H.B. \&Cetlin, P.R. Influence of die semiangle on mechanical properties of single and multiple pass drawn copper.Journal of Materials Processing Technology, 60,pp.179-182, 1996.

[2] Hwang,S.K., Baek,H.M.,Lee, J.W., Son,I.H., Im,Y.T.\&Bae,C.M. The effect of microstructure and texture evolution on mechanical properties of low carbon steel in a non-circular drawing sequence.Journal of Materials Processing Technology, 214,pp.318325, 2014.

[3] Lee, J.W.,Baek, H.M.,Hwang, S.K., Son,I.H.,Bae,C.M.\&Im,Y.T. The effect of the multi-pass non-circular drawing sequence on mechanical properties and microstructure evolution of low-carbon steel.Materials \& Design, 55,pp.898-904, 2014.

[4] Baek,H.M.,Hwang, S.K.,Joo,H.S.,Im, Y.T.,Son, I.H.\&Bae,C.M. The effect of a non-circular drawing sequence on delamination characteristics of pearlitic steel wire.Materials \& Design, 62, pp.137148, 2014.

[5] Rao,R.V.,Savsani, V.J.\&Vakharia,D.P. Teaching-learning-based optimisation: a novel method for constrained mechanical design optimisation problems.Computer-Aided Design, 43, pp.303-315, 2011.

[6] Zhou,Z.,Ong, Y.,Lim, M.\&Lee, B. Memetic algorithm using multisurrogates for computationally expensive optimization problems.Soft Computing,11,pp.957-971, 2007.

[7] Srisomporn,S.\&Bureerat, S. Geometrical design of plate-fin heat sinks using hybridization of MOEA and RSM.IEEE Transactions on Components and Packaging Technologies, 31,pp.351-360, 2008.

[8] Lophaven,S.N., Neilson,H.B.\&Sondergaard,J. DACE a MATLAB Kriging Toolbox. Technical report IMM-TR - 2002-12, KongensLyngby, Technical University of Denmark, 2002.

[9] Kazeminezhad,M.\&Taheri,A.K. Deformation inhomogeneity in flattened copper wire.Materials \& Design, 28, pp.2047-2053, 2007.

[10] Kim,S.Y.\&Im,Y.T. Three-dimensional finite element analysis of nonisothermal shape rolling.Journal of Materials Processing Technology, 127, pp.57-63, 2002

[11] Pholdee,N.\&Bureerat,S. An efficient optimum Latin hypercube sampling technique based on sequencing optimisation using simulated annealing.International Journal of Systems Science, 2014, in press.

[12] Ong,Y.S.,Zhou,Z.\&Lim,D. Curse and blessing of uncertainty in evolutionary algorithm using approximation, In: IEEE Congress on Evolutionary Computation. Vancouver, (2006) 2928-2935. 